\title{
Laboratory and febrile features after joint surgery in patients with rheumatoid arthritis treated with tocilizumab
}

\author{
M Hirao, ${ }^{1} \mathrm{~J}$ Hashimoto, ${ }^{1} \mathrm{H}$ Tsuboi, ${ }^{1}$ A Nampei, ${ }^{1} \mathrm{H}$ Nakahara, ${ }^{2} \mathrm{~N}$ Yoshio, ${ }^{2} \mathrm{~T}$ Mima, ${ }^{2}$ \\ H Yoshikawa, ${ }^{1}$ N Nishimoto ${ }^{2}$
}

${ }^{1}$ Department of Orthopaedics, Osaka University Graduate School of Medicine, Osaka, Japan; ${ }^{2}$ Laboratory of Immune Regulation, Graduate School of Frontier Biosciences, Osaka University, Osaka, Japan

Correspondence to: $\mathrm{J}$ Hashimoto, Department of Orthopaedics, Osaka University Graduate School of Medicine, 2-2 Yamadaoka, Suita City, Osaka 565-0871, Japan: junha@ort.med.osaka-u.ac.jp

Accepted 9 May 2008 Published Online First 2 June 2008 online under the BMJ Journals unlocked scheme, see http:// ard.bmj.com/info/unlocked.dtl

\author{
ABSTRACT \\ Objectives: To understand the acute phase responses to \\ surgical intervention in patients with rheumatoid arthritis \\ (RA) treated with the anti-interleukin (IL)6 receptor \\ antibody, tocilizumab.
}

Methods: In a retrospective 1:1 pair-matched casecontrol study, 22 tocilizumab-treated RA cases and 22 cases treated with conventional disease-modifying antirheumatic drugs (DMARDs) and matched for type of surgery, age and sex were evaluated for body temperature every day, and blood C-reactive protein (CRP) levels and white blood cell (WBC), neutrophil and lymphocyte counts on days $-1,1,3$ and weeks 1 and 2 after joint surgery. Safety issues were also monitored.

Results: No complications of infection or delay of wound healing occurred in either patient group. Tocilizumab partially, but significantly, suppressed the increase in body temperature on postoperative days 1 and 2, compared with DMARDs (average (SD) maximum increase in temperature was $0.45(0.1)^{\circ} \mathrm{C}$ in the tocilizumab group and $0.78(0.1)^{\circ} \mathrm{C}$ in the DMARD group; $\left.\mathrm{p}<0.01\right)$. Tocilizumab completely suppressed the increase in CRP after surgery, whereas all cases treated with DMARDs showed a significant increase of CRP at postoperative day 1 (5.5 (0.6) mg/dl; $p<0.001)$. WBC, neutrophil and lymphocyte counts showed no remarkable change after surgery, and there was no significant difference in any cell counts between the patient groups.

Conclusions: Within this small number of cases, safe operations on patients were performed during tocilizumab treatment. Tocilizumab suppressed fever and increase of CRP after surgery, whereas there was no influence on the transition in number of leukocytes. This characteristic postoperative response should be considered during tocilizumab treatment.

Postoperative surgical site infections represent a serious functional and psychological disadvantage in the course of treatment against rheumatoid arthritis (RA), although the incidence of these infrequent complications in joint surgery performed in patients with RA is approximately $1.7 \%$ to $7.2 \% .^{1-3}$ As it is necessary to detect postoperative infections as soon as possible, body temperature, level of C-reactive protein (CRP) and white blood cell (WBC) count, as well as local findings, are considered indicators of infection.

Previously published data show that the cytokine interleukin (IL) 6 is upregulated by surgical trauma and involved in the febrile response. ${ }^{23}$ The acute phase response might be diminished by blocking the synthesis of IL6 and has been reduced in genetically-modified animals that do not produce this mediator. ${ }^{45} \mathrm{CRP}$ is primarily produced in the liver in response to IL6, and CRP synthesis is enhanced synergistically by IL1 $\beta$ through induction of nuclear factor (NF) $\mathrm{kB}$, p50 and p65. ${ }^{6-8}$ In addition, IL6 positively regulates numbers of leukocytes and neutrophils, and inhibition of the IL6 receptor leads to a decrease in leukocytes. ${ }^{910}$

In recent years, use of tocilizumab, a humanised monoclonal antibody against IL6 receptor, has succeeded in achieving more effective suppression of disease activity of RA, compared to conventional disease-modifying antirheumatic drugs (DMARDs). ${ }^{11}{ }^{12}$ Based on the previous data, it is unknown whether tocilizumab also suppresses surgically-related and infection-related acute phase responses, thereby leading to possible difficulty in early diagnosis of postoperative infection due to lack of observed clinical signs and symptoms in patients treated with tocilizumab. Therefore, it is very important to understand the details of transition of data on blood tests and body temperature after joint surgery in patients with RA treated with tocilizumab. In the current study, we examined postoperative changes in body temperature and blood levels of CRP, WBCs, neutrophils and lymphocytes in patients with RA treated with tocilizumab or conventional DMARDs.

\section{PATIENTS AND METHODS \\ Patients}

A total of 22 joint surgeries in our hospital were performed on patients with RA treated with tocilizumab. In all cases, treatment with tocilizu$\mathrm{mab}$ (8 $\mathrm{mg} / \mathrm{kg}$, every 4 weeks) was continued, and surgery was performed between the administrations of tocilizumab. The most recent infusion was performed 16.1 (9.5) days (range 3 to 27 days) (mean (SD)) before operation. There was no case of postponement of infusion. All surgeries were performed under general anaesthesia. Operations included shoulder arthroplasty $(n=1)$, total elbow arthroplasty (TEA) $(n=1)$, total hip arthroplasty (THA) ( $\mathrm{n}=1)$, total knee arthroplasty (TKA) $(n=9)$, total ankle arthroplasty (TAA) $(n=3)$, foot surgery $(n=5)$ and hand surgery $(n=2)$.

Retrospectively, we studied these 22 cases (tocilizumab group) and compared them to 22 operations in patients with RA who received nonbiological medication (methotrexate: 6 cases; salazosulfapyridine: 10 cases, bucillamine: 3 cases, D-penicillamine: 1 case, prednisolone: 17 cases) 
and who underwent joint surgery. For matching of cases, patients were selected with a 1:1 pair-matched procedure, according to age (range \pm 5 years) and type and site of surgery.

\section{Body temperature}

Body temperature was measured at least three times daily (at $9.00 \mathrm{am}, 2.00 \mathrm{pm}$ and $7.00 \mathrm{pm}$ ) during hospitalisation. All measurements were performed for $5 \mathrm{~min}$ at the external ear canal on the same side. For analysis, we used the peak body temperature at 1 day before surgery, during 1 and 2 days after surgery, 1 week after surgery (week 1) and 2 weeks after surgery (week 2). Expected fever spikes after surgery in the tocilizumab and the DMARD groups were evaluated by change in body temperature, as compared before the operation.

\section{Blood samples}

Venous blood samples were obtained before surgery, 1 and 3 days after surgery, 1 week after surgery and 2 weeks after surgery. Samples were collected in EDTA tubes and analysed for WBC counts and segmentation of WBCs (neutrophils and lymphocytes). The change in WBCs, neutrophils and lymphocytes after surgery was evaluated by the percentage changes in these parameters, compared with preoperative values. CRP levels were measured in serum. In our hospital, the normal reference value for CRP was $<0.2 \mathrm{mg} / \mathrm{dl}$.

\section{Statistical analysis}

All data are expressed as mean (standard error of the mean (SEM)). Differences between the groups were assessed by a post hoc test using SPSS statistical analysis software (SPSS V. 15.0J; SPSS, Chicago, Illinois, USA). A p value of $<0.05$ was considered statistically significant.

\section{RESULTS}

\section{Postoperative complications}

No complications of superficial infections, deep infections, or delay in wound healing were observed in either group of cases.

\section{Body temperature}

As shown in table 1, there was no significant difference in preoperative body temperature between the tocilizumab and the DMARD groups. Both groups showed significant increases in body temperature, as compared with that of the preoperative day (fig 1). The expected increase in body temperature following surgery was significantly suppressed in the tocilizumab group (fig 1). Average maximum increase in body temperature was $0.78(0.1)^{\circ} \mathrm{C}$ in the DMARD group and 0.45 $(0.1)^{\circ} \mathrm{C}$ in the tocilizumab group.

\section{CRP}

Preoperative CRP levels in the tocilizumab group were negative in all cases except one, who showed $0.3 \mathrm{mg} C R P / d l$. All cases in the DMARD group showed positive CRP values, and the mean (SD) value was $3.1(0.6) \mathrm{mg} / \mathrm{dl}$ (table 1$)$. After surgery, 18 of the 22 tocilizumab-treated cases showed no postoperative increase in CRP, whereas the remaining 4 cases showed an increase ranging from 0.1 to $1.0 \mathrm{mg} / \mathrm{dl}$. In the DMARD group, all cases showed a significant increase (day 1: 5.5 (0.6) mg/dl; 1 week after surgery: $2.9(0.5) \mathrm{mg} / \mathrm{dl} ; 2$ weeks after surgery: 2.2 (0.5) $\mathrm{mg} / \mathrm{dl}$ ).

\section{WBC counts}

As shown in table 1, there was no significant difference in preoperative numbers of WBCs, neutrophils, or lymphocytes between the tocilizumab and the DMARD groups. These cell numbers showed no remarkable changes after surgery in either group. Additionally, no significant difference between the groups was observed in these parameters after surgery. Therefore, cell numbers after surgery were not affected by tocilizumab treatment.

\section{DISCUSSION}

An important issue in anti-inflammatory cytokine therapy is the possible risk of infection, because a recent study showed that tumour necrosis factor (TNF) $\alpha$ antagonists increase the risk of infection for musculocutaneous lesions. ${ }^{13}$ Reports on small studies involving 12 to 16 cases of joint surgery in patients with RA treated with TNF $\alpha$ antagonists, however, demonstrated no increase in the incidence of postoperative infection. ${ }^{14} 15$

Our hospital's experience with 22 cases of joint surgery in patients with RA treated with tocilizumab also showed no postoperative surgical site infections. Although IL6 knock-out (IL6KO) mice display significantly delayed cutaneous wound healing compared to wild type mice, ${ }^{16}{ }^{17}$ we observed no delay in postoperative wound healing in patients with RA treated with tocilizumab. Because the reported incidence of postoperative infection is around $1.7 \%$ to $7.2 \%,{ }^{1-3}$ the lack of statistical power due to the small number of patients in our study made it impossible to reach a conclusive result as to the influence of tocilizumab treatment on the risk of postoperative infection. However, our experience that there were no postoperative infections in either tocilizumab or DMARD groups suggests the safety of joint surgery in patients with RA during tocilizumab treatment.

High-level production of proinflammatory cytokines including IL6 in the inflamed synovium has a pathological role in systemic manifestations of RA, such as fatigue, fever and laboratory changes. ${ }^{18}$ After surgical treatment, immune cells such as macrophages and neutrophils or other cells such as fibroblasts and endothelial cells are activated locally at the surgical site by destruction of tissues, resulting in increased local and serum levels of IL6. ${ }^{219}$ Therefore, it is plausible that inhibition of IL6 suppresses the systemic inflammatory manifestations of RA and surgery. Indeed, the postoperative fever spike was partially, but significantly, suppressed by tocilizumab treatment, as compared with DMARD treatment in the present study.

IL6 stimulates hepatocytes to produce acute phase proteins such as CRP, fibrinogen, $\alpha 1$-anti-trypsin and serum amyloid A, and simultaneously suppresses albumin production. Previous reports have shown that the increase in CRP and serum amyloid A were normalised after tocilizumab treatment and the decrease in albumin was alleviated after tocilizumab treatment. ${ }^{10-12}{ }^{20}$ In the current study, the preoperative CRP values were normal $(<0.2)$ in 21 out of 22 cases in the tocilizumab group. Furthermore, a postoperative increase in CRP was not observed in the tocilizumab group, whereas in the DMARD group the mean CRP level changed from a preoperative level of $3.1 \mathrm{mg} / \mathrm{dl}$ to a maximum level of $5.5 \mathrm{mg} / \mathrm{dl} 1$ day after surgery. This finding clearly indicates that tocilizumab suppressed not only an RA-related increase of CRP, but also a surgery-related increase in CRP. Tocilizumab treatment also may suppress infection-related symptoms after surgery. Although no infection was observed in the patients of this study, we have experienced 

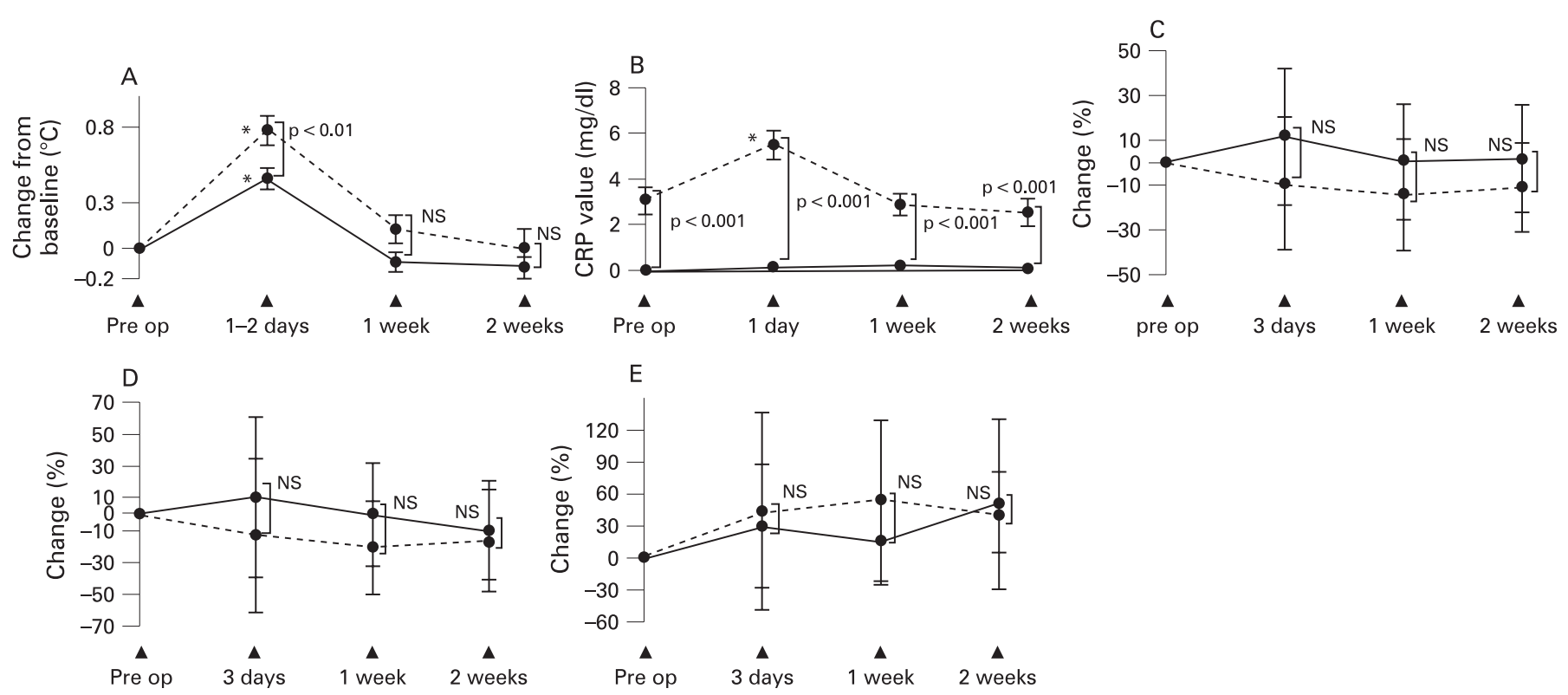

Figure 1 Changes in body temperature, C-reactive protein (CRP) levels and numbers of white blood cells (WBCs; neutrophils and lymphocytes) after surgery. Values are mean (SD). * Significant difference, as compared to values before surgery. NS, no significant difference between the two groups. Dotted lines: disease-modifying antirheumatic drug (DMARD) group. Unbroken lines: tocilizumab group. A. Graph shows the change in body temperature, as compared before surgery. $p$ : $p$ Value for the difference between the DMARD group and the tocilizumab group. B. Graph shows the absolute value of CRP level. p: p Value for the difference between the two groups. C. Graph shows the percentage change in total number of WBCs. D. Graph shows the percentage change in numbers of neutrophils. E. Graph shows the percentage change in numbers of lymphocytes.

some tocilizumab-treated patients with pneumonia without operation. As long as the infection was not severe, serum level of CRP hardly increased while the WBC count did increase. When the patients had severe pneumonia, they showed an increase in CRP level and WBC count (data not shown). This may be explained by the balance between the blood concentration of IL6 and tocilizumab, which competitively binds to IL6 receptor. Severe infection induces a high amount of IL6, which cannot be blocked with usual doses of tocilizumab, resulting in the increase in CRP. Similarly, if the surgical invasion is more severe, CRP would be expected to increase even under tocilizumab treatment. In addition, since the WBC count is less influenced by IL6 than CRP, an increase in WBC count could be a useful sign for possible infection. In the DMARD group, the level of CRP was restored to the basal level within 1 week after surgery, suggesting that surgery-induced inflammation resolves within 1 week in the absence of infection.

A dose-dependent reduction in the neutrophil count following treatment with tocilizumab was reported in patients with RA. ${ }^{11}{ }^{12}$ In the present study, preoperative absolute numbers of

Table 1 Characteristics of the cases before surgery

\begin{tabular}{lccl}
\hline Parameter & $\begin{array}{l}\text { No. treated with } \\
\text { DMARDs }\end{array}$ & $\begin{array}{l}\text { No. treated with } \\
\text { tocilizumab }\end{array}$ & p Value \\
\hline Body temperature $\left({ }^{\circ} \mathrm{C}\right)$ & $36.5(0.3)$ & $36.6(0.2)$ & NS \\
CRP $(\mathrm{mg} / \mathrm{dl})$ & $3.1(0.6)$ & $0.02(0.02)$ & $<0.001$ \\
WBCs/dl & $8144(3229)$ & $7267(2757)$ & $\mathrm{NS}$ \\
Neutrophils/ml & $6716(2973)$ & $5424(2545)$ & $\mathrm{NS}$ \\
Lymphocytes/ml & $978(413)$ & $1384(706)$ & $\mathrm{NS}$ \\
DAS28 (CRP) score & $4.4(0.9)$ & $2.7(0.7)$ & $<0.001$ \\
Prednisolone (mg/day) & $7.0(5.1)$ & $6.5(5.1)$ & $\mathrm{NS}$ \\
\hline
\end{tabular}

Values are mean (SD). $p$ Value is for the difference between the DMARD group and the tocilizumab group.

CRP, C-reactive protein; DAS28, 28-joint Disease Activity Score; DMARD, diseasemodifying antirheumatic drug; NS, not significant; WBC, white blood cell.
WBCs and neutrophils in the tocilizumab group were smaller, but not significantly smaller, than those in the DMARD group. By contrast, preoperative absolute numbers of lymphocytes in the tocilizumab group were larger, but not significantly larger, compared to the DMARD group. No significant postoperative changes in leukocytes, neutrophils, or lymphocytes were observed in either group, and additionally no significant differences between the two groups were seen at any time after surgery. These data suggest that regulation of the increase in leukocytes may depend on not only IL6 but also other cytokines such as granulocyte colony stimulating factor (G-CSF) and granulocyte-macrophage colony stimulating factor (GM-CSF).

Our data suggest the possibility that tocilizumab might mask the infection-induced increase in CRP and minimise the infection-induced increase in body temperature after orthopaedic surgery in patients with RA. In other words, a small postoperative increase in CRP in patients with RA may be an important sign of the occurrence of non-arthritic types of inflammation, such as postoperative infections. In cases of postoperative increases in CRP in tocilizumab recipients, signs of inflammation in other organs, such as the respiratory system, as well as signs of infection at the surgical site, should be checked. A WBC count may be helpful in knowing whether a concurrent infection is occurring during tocilizumab therapy. Furthermore, serum IL6 should be a good marker for the severity of inflammation because tocilizumab blocks not IL6 itself but the IL6 receptor. ${ }^{45}$

In conclusion, although the current study evaluated only 22 cases of orthopaedic surgery in patients with RA treated with tocilizumab and 22 cases of orthopaedic surgery in patients with RA treated with DMARDs, no complications of superficial or deep infection or delay in wound healing after orthopaedic surgery were observed. Additionally, we demonstrated that the increase in CRP was completely suppressed and the rise in body 
temperature was partially suppressed after joint surgery in tocilizumab-treated patients with RA, whereas tocilizumab had no significant influence on the number of leukocytes. Considering these characteristic postoperative responses in patients with RA treated with tocilizumab, we should carefully perform orthopaedic surgery on the patients.

Acknowledgements: We would like to thank Chugai Pharmaceuticals for providing tocilizumab.

Competing interests: NN and TM have served as consultants to and/or received honoraria from Chugai Pharmaceutical, the manufacture of tocilizumab.

Ethics approval: Ethics approval was obtained.

\section{REFERENCES}

1. Matewski D, Szymkowiak E, Gumański R, Puchala J, Sniegowski M. Outcome of total hip replacement in rheumatic arthritis. Ortop Traumatol Rehabil 2005:6:633-8.

2. Karel W, Smet LD. The Kudo total elbow arthroplasty in patients with rheumatoid arthritis. J Shoulder Elbow Surg 2004;13:542-7.

3. Abhilash J, Melinda W, Cathy B, Jagdeep N. Influence of steroids and methotrexate on wound complications after elective rheumatoid hand and wrist surgery. J Hand Surg 2002;27A:449-55.

4. Nishimoto N, Yoshizaki K, Tagoh H, Monden M, Kishimoto S, Hirano T, et al. Elevation of serum interleukin 6 prior to acute phase proteins on the inflammation by surgical operation. Clin Immunol Immunopathol 1989;50:399-401.

5. Ohzato H, Yoshizaki K, Nishimoto N, Ogata A, Tagoh H, Monden M, et al. Interleukin6 as a new indicator of inflammatory status: detection of serum levels of interleukin-6 and C-reactive protein after surgery. Surgery 1992:111:201-9.

6. Leon LR. Invited review: cytokine regulation of fever: studies using gene knockout mice. J Appl Physiol 2002; 92:2648-55.

7. De Jongh RF, Visser KC, Booij LH, De Jongh KL, Vincken P, Meert TF. Interleukin-6 and perioperative thermoregulation and HPA-axis activation. Cytokine 2003;21:248-56.

8. Gauldie J, Richards C, Harnish D, Lansdorp P, Baumann H. Interferon $\beta$ 2/B-cell stimulatory factor type 2 shares identity with monocyte-derived hepatocytestimulating factor and regulates the major acute phase protein response in liver cells. Proc Natl Acad Sci USA 1987;84:7251-5.
9. Mackiewicz A, Speroff T, Ganapathi MK, Kushner I. Effects of cytokine combinations on acute phase protein production in two human hepatoma cell lines. J Immunol 1991;146:3032-7.

10. Agrawal A, Cha-Molstad H, Samols D, Kushner I. Overexpressed nuclear factor- $\kappa B$ can participate in endogenous $\mathrm{C}$-reactive protein induction, and enhances the effect of C/EBP beta and signal transducer and activator of trabscription-3. Immunology 2003;108:539-47.

11. van Gameren $\mathbf{M M}$, Willemse $\mathrm{PH}$, Mulder $\mathrm{NH}$, Limburg $\mathrm{PC}$, Groen $\mathrm{HJ}$, Vellenga $\mathrm{E}$, et al. Effects of recombinant human interleukin-6 in cancer patients: A phase I- II study. Blood 1994;84:1434-41.

12. Nishimoto N, Sasai M, Shima $Y$, Nakagawa M, Matsumoto T, Shirai T, et al Improvement in Castleman's disease by humanized anti-interleukin-6 receptor antibody therapy. Blood 2000;95:56-61.

13. Nishimoto N, Yoshizaki K, Miyasaka N, Yamamoto K, Kawai S, Takeuchi T, et al. Treatment of rheumatoid arthritis with humanized anti-interleukin-6 receptor antibody: a multicenter, double-blind, placebo-controlled trial. Arthritis Rheum 2004;50:1761-9.

14. Maini RN, Taylor PC, Szechinski J, Pavelka K, Bröll J, Balint G, et al. Double-blind randomized controlled clinical trial of the interleukin-6 receptor antagonist, tocilizumab, in European patients with rheumatoid arthritis who had an incomplete response to methotrexate. Arthritis Rheum 2006:54:2817-29.

15. Strangfeld A, Listing J. Infection and musculoskeletal conditions: bacterial and opportunistic infections during anti-TNF therapy. Best Pract Res Clin Rheumatol 2006;20:1181-95

16. Shergy WJ, Philips RM, Hunt RE, Hernandez J. Infliximb and its impact on surgical outcomes in rheumatoid arthritis. Ann Theum Dis 2005;64(Suppl III):465.

17. Talwalker SC, Grennan DM, Gray J, Johnson P, Hayton MJ. Tumor necrosis factor $\alpha$ antagonists and early postoperative complications in patients with inflammatory joint disease undergoing elective orthopedic surgery. Ann Rheum Dis 2005:64:650-1.

18. Gallucci RM, Sugawara T, Yucesoy B, Berryann K, Simeonova PP, Matheson JM, et al. Interleukin-6 treatment augments cutaneous wound healing in immunosuppressed mice. J Interferon Cytokine Res 2001;21:603-9.

19. Lin Z0, Kondo T, Ishida Y, Takayasu T, Mukaida N. Essential involvement of IL-6 in the skin wound-healing process as evidenced by delayed wound healing in IL-6deficient mice. J Leukoc Biol 2003;73:713-21.

20. Weber J, Yang JC, Topalian SL, Parkinson DR, Schwartzentruber DS, Ettinghausen $\mathrm{SE}$, et al. Phase I trial of subcutaneous interleukin- 6 in patients with advanced malignancies. J Clin Oncol 1993;11:499-506. 